\title{
Electronic cigarettes and thirdhand tobacco smoke: two emerging health care challenges for the primary care provider
}

This article was published in the following Dove Press journal:

International Journal of General Medicine

28 January 2011

Number of times this article has been viewed

\author{
Ware G Kuschner \\ Sunayana Reddy \\ Nidhi Mehrotra \\ Harman S Paintal \\ Division of Pulmonary and Critical \\ Care Medicine, Stanford University \\ School of Medicine, Palo Alto, \\ CA, USA
}

\begin{abstract}
Primary care providers should be aware of two new developments in nicotine addiction and smoking cessation: 1) the emergence of a novel nicotine delivery system known as the electronic (e-) cigarette; and 2) new reports of residual environmental nicotine and other biopersistent toxicants found in cigarette smoke, recently described as "thirdhand smoke". The purpose of this article is to provide a clinician-friendly introduction to these two emerging issues so that clinicians are well prepared to counsel smokers about newly recognized health concerns relevant to tobacco use. E-cigarettes are battery powered devices that convert nicotine into a vapor that can be inhaled. The World Health Organization has termed these devices electronic nicotine delivery systems (ENDS). The vapors from ENDS are complex mixtures of chemicals, not pure nicotine. It is unknown whether inhalation of the complex mixture of chemicals found in ENDS vapors is safe. There is no evidence that e-cigarettes are effective treatment for nicotine addiction. ENDS are not approved as smoking cessation devices. Primary care givers should anticipate being questioned by patients about the advisability of using e-cigarettes as a smoking cessation device. The term thirdhand smoke first appeared in the medical literature in 2009 when investigators introduced the term to describe residual tobacco smoke contamination that remains after the cigarette is extinguished. Thirdhand smoke is a hazardous exposure resulting from cigarette smoke residue that accumulates in cars, homes, and other indoor spaces. Tobaccoderived toxicants can react to form potent cancer causing compounds. Exposure to thirdhand smoke can occur through the skin, by breathing, and by ingestion long after smoke has cleared from a room. Counseling patients about the hazards of thirdhand smoke may provide additional motivation to quit smoking.
\end{abstract}

Keywords: e-cigarette, nicotine, tobacco, thirdhand smoke, electronic nicotine delivery systems

\section{Background}

Tobacco use is the leading cause of preventable illness in the United States, responsible for more than 443,000 deaths annually. ${ }^{1}$ Since the 1964 US Surgeon General's report announcing cigarette smoking to be harmful, concerns about tobacco's deadly toll have become widespread and efforts to curb smoking have progressively intensified. ${ }^{2}$ Once thought to be a "habit" or "custom", cigarette smoking and other forms of tobacco use are now widely recognized as behaviors driven by nicotine addiction. It is addiction to nicotine that compels many smokers to continue to use tobacco products even when they appreciate its harmful effects and wish to quit. ${ }^{3,4}$

The cigarette is an effective instrument for delivering nicotine to the body. Burned tobacco produces vaporized nicotine which is easily entrained into the lungs. 
Within 10 to 15 seconds of puffing on a cigarette, nicotine is absorbed into the bloodstream of smokers and travels to the brain where it acts on nicotinic cholinergic receptors to produce a range of gratifying effects. ${ }^{5}$ The release of a variety of neurotransmitters results in neural activation, producing effects such as pleasure, appetite suppression, arousal, cognitive enhancement, relaxation, and reduction in anxiety. ${ }^{6-8}$

For most smokers, an attempt to achieve long-term abstinence results in failure. To improve outcomes, medications, counseling, and education have become the standard of care for smoking cessation treatment. Medications that have been shown to improve smoking cessation rates include nicotine replacement therapies, available as gum, patches, an inhaler, a nasal spray, a lozenge, and also include varenicline and bupropion. ${ }^{9-12}$

Public policy measures to promote smoking cessation and parallel measures to reduce smoking initiation have had major positive public health effects. Health care organizations now provide structured smoking cessation services, recognizing the value of this health maintenance intervention. Indeed, smoking cessation treatment has become a service that primary care providers are widely expected to deliver, which may be tracked as a quality measure in many health care organizations. ${ }^{13-15}$

Despite the expectation that primary care providers encourage and orchestrate the smoking cessation efforts of their patients, there may be persistent uncertainty regarding the physician's precise role in managing the nicotine addicted patient. Part of this uncertainty results from the belief that smoking is now globally appreciated to be harmful and that delivering education about the hazards of smoking is not the best use of limited time during an office visit. The widespread availability of smoking cessation "quitlines" which provide free counseling and education by telephone in almost all states in the United States has also been an important development which provides opportunities for education and professional counseling without direct involvement of a physician. ${ }^{16-18}$ Additionally, some smoking cessation medications (nicotine replacement drugs) are available over-the-counter, so that the smoker who is motivated to quit smoking can obtain pharmacologic treatment without a visit to a physician. Nevertheless, despite the availability of smoking cessation treatment without physician intervention, primary care physician involvement in smoking cessation efforts remains an expectation of patients and other stakeholders pursuing optimal preventive health care and clinical outcomes. ${ }^{14}$
Two new developments in nicotine addiction and smoking cessation add some measure of complexity to the clinician's important role in directing patients' smoking cessation efforts: the emergence of a novel nicotine delivery system known as the electronic (e-) cigarette; and new reports of the hazards of residual environmental nicotine and other biopersistent toxicants found in cigarette smoke, recently described as "thirdhand smoke". ${ }^{19}$ The remainder of the article provides a concise, clinician-friendly update on the e-cigarette and thirdhand smoke. The goal of this review is to provide clinicians with useful new information relevant to nicotine addiction, tobacco use, and smoking cessation. This information can be incorporated into patient counseling that promotes safe and effective smoking cessation strategies.

\section{Electronic cigarettes - an untested nicotine delivery device}

Electronic cigarettes are battery powered devices that convert nicotine containing liquid into a vapor that can be inhaled. The World Health Organization has termed these devices electronic nicotine delivery systems (ENDS). ${ }^{20}$ We use the terms electronic cigarette, e-cigarette, and ENDS interchangeably in this review, underscoring the fact that clinicians may encounter any of these terms in the growing literature on this novel nicotine delivery system. The term "vaping" has emerged to describe use of the ENDS; that is, the inhalation of e-cigarette vapors. ${ }^{21,22}$

While multiple companies now manufacture ENDS, the basic design is generally similar. E-cigarettes consist of a plastic tube, an electronic heating element, a liquid nicotine cartridge, and a lithium battery and atomization chamber with a membrane to suspend ingredients. ${ }^{23}$ Some e-cigarettes contain a light emitting diode in the tip which illuminates when the user inhales (or "vapes"), giving the appearance of the burning end of a conventional cigarette. Chemicals introduced into the liquid vehicle produce aromas and flavors of tobacco, chocolate, mint, fruit, and coffee. ${ }^{24}$

ENDS were developed with the goal of mimicking the efficient nicotine delivery system of a conventional cigarette without the significant harmful effects of tobacco smoke. They are often marketed as the healthier version of the conventional cigarette. The device aerosolizes nicotine so that it is readily entrained into the respiratory tract and then enters the bloodstream, resulting in a near instantaneous nicotine reward in the central nervous system. Propylene glycol - the chemical used to generate artificial "smoke" for theatrical productions - is added to the liquid vehicle to stimulate the appearance of using a "real" cigarette. The concentration of nicotine varies both 
across different manufacturers and within the same brand. Cartridges vary in their nicotine content from no nicotine to 16 to $18 \mathrm{mg}$ per cartridge. ${ }^{20}$ The cost of an e-cigarette starter kit can range from US\$40 to 120 with additional cartridges costing a few dollars each. After the initial investment in the starter kit, the recurrent daily costs (of cartridges) are somewhat more than conventional cigarettes.

Information on the pharmacology, toxicology, and safety of e-cigarettes is limited. Accessible information provides details about the presence of nicotine (including cotinine), tobacco specific impurities (anabasine, myosmine, $\beta$-nicotyrine), propylene glycol (rarely diethylene glycol), and tobacco specific nitrosamines (which include carcinogens) in commonly available brands of electronic cigarettes. ${ }^{24-26}$ Some of the available data have been gathered from research sponsored by manufacturing companies of these devices. ${ }^{27}$ No research has been conducted to test the efficacy of these cigarettes as smoking cessation aids. ${ }^{28}$ The marketing and sales of these products have not been followed by post-marketing studies looking at health effects, probably because the products are not regulated as drug delivery devices in most countries. Despite this knowledge gap, consumer interest in ENDS is growing rapidly and concerns about their unregulated use are intensifying.

A recent study found that the effort required (vacuum desired) to smoke an e-cigarette is higher than that for conventional cigarettes. ${ }^{29}$ This effect was accentuated after a few puffs resulting in nonuniform nicotine delivery, leading the authors to question ENDS as effective vehicles of nicotine into the patient's lungs. Surveys have shown that while consumers buy electronic cigarettes for a variety of reasons (such as trying to quit smoking, preventing exposure to smoke, being allowed to smoke in smoke free zones, better flavor), they remain concerned about the potential hazards associated with their use. ${ }^{30}$ One study has shown decreased desire to smoke with the use of e-cigarettes after overnight abstinence, when compared with a placebo or regular cigarettes, and an effect similar to a nicotine inhaler. ${ }^{31}$ However, ENDS as a treatment for nicotine addiction, or as promoting long-term abstinence from tobacco, have not been formally investigated.

Claims that ENDS can aid in smoking cessation have been scrutinized by the US Food and Drug Administration (FDA). ${ }^{32}$ The FDA's position is that ENDS meet the definition of a drug-delivery device under the Food, Drug, and Cosmetic Act and should be regulated accordingly; just as nicotine inhalers are regulated. Thus manufacturers would be required to submit an application for evaluation and approval of their devices before they could be marketed. In September 2010, the FDA sent letters to 5 manufacturers of ENDS, notifying them that they were in violation of federal law by making claims that their products could help smokers quit. On December 6, 2010, a three-judge appellate panel from the US Court of Appeals for the District of Columbia Circuit ruled that the FDA lacks authority to regulate electronic cigarettes as drugs or devices, thus upholding a lower court decision. The US Court of Appeals ruled that the FDA can only regulate ENDS as tobacco products. On December 20, 2010, the FDA filed a petition asking the US Court of Appeals in its entirety to conduct a hearing to review the agency's appeal in its effort to regulate electronic cigarettes as devices delivering drugs. A decision is pending. Under the new Family Smoking Prevention and Tobacco Control Act passed by Congress in 2009 , the FDA has the authority to regulate tobacco products and therefore may have the authority to regulate ENDS, but not ban them, if this most recent court ruling holds. Through regulation, ENDS have essentially been banned in Australia, Canada, Singapore, and Brazil because of the lack of data about their safety or efficacy. ${ }^{20}$

\section{Counseling smokers and "vapers" about e-cigarettes}

What should the primary care provider tell the patient who smokes tobacco cigarettes, wants to quit, and is interested in e-cigarettes, or is already using e-cigarettes? What should clinicians tell the patient who may be using e-cigarettes in spaces where tobacco cigarettes are banned and who has no interest in quitting smoking?

Clinicians should counsel patients based on their reported tobacco use and their e-cigarette use behaviors and interests. Social, demographic, and health considerations should also inform the education and counseling process. We list below a set of quick facts and prudent talking points that clinicians can utilize to facilitate healthy behavioral change and reinforce prudent health habits:

- ENDS are not FDA approved as quit smoking devices.

- The vapors from e-cigarettes are complex mixtures of chemicals, not pure nicotine. Whether inhalation of the complex mixture of chemicals in ENDS vapors is safe is unknown.

- There is no evidence that ENDS help smokers to quit smoking.

- There is increasing resistance to the use of ENDS in public places and outright bans in a growing number of states.

- The promotion of ENDS may communicate a message to children and adolescents that "vaping" is harmless, 
inadvertently increasing the risk of nicotine addiction and tobacco use in a vulnerable population.

- FDA-approved treatments for smoking cessation, proven safe and effective, are available.

- Long-term abstinence from tobacco use is a goal that has been achieved by millions of people. Almost half of ever smokers alive today are former smokers.

- Until more information about ENDS becomes available, use of e-cigarettes cannot be recommended.

- Smoking cessation medications, conventional counseling strategies, including the 5 major steps to intervention "The 5 As": (ask, advise, assess, assist, arrange), and telephone quitlines should be offered to patients who smoke and who wish to quit.

- Physicians can use information freely available on the web to guide their practice (http://www.surgeongeneral. gov/tobacco/tobaqrg.htm).

- 1-800-QUIT-NOW: The US Department of Health and Human Services sponsors this national telephone quitline. The toll-free number is a single access link to the national network of tobacco cessation quitlines. http:// www.smokefree.gov.

\section{Thirdhand smoke - the hazards of biopersistent cigarette smoke residue}

The term thirdhand smoke first appeared in the medical literature in 2009 when investigators defined it as residual tobacco smoke contamination that remains after the cigarette is extinguished. ${ }^{19}$ Anecdotal evidence suggests that nicotinejust one constituent of tobacco smoke - may persist in ceiling tiles for up to 30 years. ${ }^{33} \mathrm{~A}$ recent study found that nicotine persists in homes previously occupied by smokers and that nonsmokers who move into these homes have elevated levels of nicotine on their skin and in body fluids. ${ }^{34}$

Thirdhand smoke should be distinguished from second-hand smoke. Second-hand smoke refers to a nonsmoker's exposure to the airborne products of tobacco combustion, typically in close temporal and physical proximity to cigarette smoking. In contrast, thirdhand smoke exposure may occur long after cigarette smoking has ceased. Particulates from cigarette smoke deposit on indoor surfaces and persist, resulting in exposures that may occur well into the future. ${ }^{35,36}$ Thirdhand smoke exposure is a risk of special concern to children because they may crawl or play on contaminated surfaces. There is no evidence that exposure to thirdhand smoke can be reduced or eliminated by increasing the rate of air exchange in an indoor environment through fans, open windows, or air conditioners after contaminants have deposited on surfaces. Parents who believe in the concept of thirdhand smoke exposure are more likely to enforce smoking bans inside the house. ${ }^{19}$

The cigarette smoke residue that persists on surfaces may be toxic. Nicotine, which is not a carcinogen, can deposit on surfaces and then react with ambient nitrous acid to produce tobacco specific nitrosamines (TSNAs) which include carcinogenic compounds. The main indoor sources of ambient nitrous oxide are unvented gas appliances. ${ }^{37,38}$

In an experimental model using cellulose as a model indoor material, a greater than 10-fold increase of surfacebound TSNA was generated when sorbed second-hand smoke was exposed to nitrous acid for 3 hours. ${ }^{39}$ In this experiment, second-hand smoke cellulose substrates were collected in a room-sized $18-\mathrm{m}^{3}$ environmental chamber with low background concentrations of airborne contaminants. Tobacco smoke was generated in a chamber by using a smoking machine. Nine cigarettes were smoked in 3 hours. A cellulose substrate was the medium on which nicotine from tobacco smoke and nitrous acid reacted to produce TSNA.

Substantial TSNA levels were also measured in the passenger compartment of a light duty pick-up truck in which the driver routinely smoked while commuting. ${ }^{40}$ In both models, the investigators detected a hazardous TSNA, 1-(N-methyl-N-nitrosamino)-1-(3-pyridinyl)-4-butanal, which is absent in freshly emitted tobacco smoke. ${ }^{41}$ Other potent carcinogens were also detected, including 4-(methylnitrosamino)-1-(3-pyridinyl)-1-butanone and $\mathrm{N}$-nitroso nornicotine. ${ }^{39}$ Investigators concluded that given the rapid sorption and persistence of high levels of nicotine on indoor surfaces, this newly identified process results in continuing exposure to hazardous TSNAs long after smoke is cleared. Dermal exposure, dust inhalation, and ingestion are all potential routes of exposure to these toxic compounds.

Nicotine deposits readily on surfaces and gradually outgases. Simulation of intense indoor smoking found no threshold for the adsorption of nicotine to wallboard. Accordingly, standard indoor domestic walls are likely to serve as high capacitance reservoirs for nicotine which can then be converted to TSNAs as described above. ${ }^{42,43}$

Although data are limited, it appears that the potential hazards of thirdhand smoke exposure persist long after smoking has ceased. Toxic exposures might even persist for years. To eliminate the health risks associated with thirdhand smoke, it would be necessary to remove the furniture, carpets, drapes, curtains, and wallboards of contaminated spaces.

In June 2010, the University of California Tobacco-Related Disease Research Program announced a US\$3.75 million 
initiative to study the problem of thirdhand smoke. Goals include identifying the spectrum of toxicants, routes of exposure, health effects and the magnitude of exposure in occupational and domestic settings. ${ }^{44}$

\section{Incorporating knowledge about thirdhand smoke into clinical practice}

Thirdhand smoke is an emerging health concern. The magnitude of the public health threat presented by this exposure is not fully known. Nevertheless, general comments about concerns presented by thirdhand smoke can be introduced into conversations with patients about the health hazards of smoking and their effects on bystanders who do not smoke. As in all conversations with patients and their family members about tobacco use, the message should be relevant and targeted. Those patients who smoke in their home and are concerned about the welfare of nonsmokers who live in or visit the home may be most responsive to discussions about thirdhand smoke. Currently, many smokers believe that "airing out" an indoor space reduces the risk for second-hand smoke exposure and therefore renders the space clean and healthy. However, the message from research on thirdhand smoke is clear: health hazards attributable to cigarette smoking persist long after the cigarette is extinguished.

Cleansers that dissolve nicotine into water must be acidic (eg, vinegar). Most soaps are alkaline and will not effectively remove nicotine residue. While it may be possible to remove nicotine from smooth surfaces with an acidic cleaning product, removing thirdhand smoke from carpet may be impossible. ${ }^{45}$

We list below a set of quick facts and talking points that center on thirdhand smoke, which clinicians can use to facilitate healthy behavioral change and to reinforce prudent health habits:

- Thirdhand smoke is a hazardous exposure resulting from cigarette smoke residue that accumulates in cars, homes, and other indoor spaces.

- Toxicants that deposit indoors may persist indefinitely.

- Tobacco-derived toxicants can react to form potent cancer-causing compounds.

- Exposure to thirdhand smoke can occur through the skin, by breathing, and by ingestion long after smoke has been cleared out of a room.

- Children who climb on furniture, crawl and play on floors, may be especially susceptible to thirdhand smoke.

- It appears there is no safe way for nonsmokers to cohabitate with smokers who smoke indoors.

\section{Summary}

The e-cigarette is a novel nicotine delivery device, which has some appealing features and is gaining widespread interest. However, a paucity of knowledge about e-cigarettes or electronic nicotine delivery systems (ENDS) and concerns about their safety and public health impact have raised important concerns about their use. ENDS are widely available, but are not FDA approved as quit smoking devices.

Thirdhand smoke is a newly described health hazard that results from cigarette smoking. Thirdhand smoke is a hazardous exposure resulting from cigarette smoke residue, which accumulates indoors and in the passenger compartments of motor vehicles. Exposure to thirdhand smoke can occur long after airborne products of combustion (ie, smoke) have been cleared from a room.

Clinicians should incorporate insights about ENDS and thirdhand smoke into conversations with patients who smoke in order to facilitate healthy behavioral changes including smoking cessation.

\section{Disclosure}

The authors declare no conflicts of interest.

\section{References}

1. Centers for Disease Control and Prevention. Tobacco Use. Targeting the Nation's Leading Killer: At A Glance 2010. http://www.cdc. gov/chronicdisease/resources/publications/aag/osh.htm. Accessed January 7, 2011.

2. Smoking and Health: Report of the Advisory Committee to the Surgeon General of the Public Health Service. Public Health Service. Office of the Surgeon General. Public Health Service Publication No. 1103 http://profiles.nlm.nih.gov/NN/B/B/M/Q. Accessed January 7, 2011.

3. Portugal GS, Gould TJ. Genetic variability in nicotinic acetylcholine receptors and nicotine addiction: converging evidence from human and animal research. Behav Brain Res. 2008;193(1):1-16.

4. Mobascher A, Rujescu D, Mittelstrass K, et al. Association of a variant in the muscarinic acetylcholine receptor 2 gene (CHRM2) with nicotine addiction. Am J Med Genet B Neuropsychiatr Genet. 2010; 153B(2):684-690.

5. Berridge MS, Apana SM, Nagano KK, Berridge CE, Leisure GP, Boswell MV. Smoking produces rapid rise of [11C] nicotine in human brain. Psychopharmacology (Berl). 2010;209(4):383-394.

6. Metz CN, Gregersen PK, Malhotra AK. Metabolism and biochemical effects of nicotine for primary care providers. Med Clin North Am. 2004;88(6):1399-1413, ix.

7. Chelland Campbell S, Moffatt RJ, Stamford BA. Smoking and smoking cessation - the relationship between cardiovascular disease and lipoprotein metabolism: a review. Atherosclerosis. 2008;201(2):225-235.

8. Cosci F, Knuts IJ, Abrams K, Griez EJ, Schruers KR. Cigarette smoking and panic: a critical review of the literature. J Clin Psychiatry. 2010; 71(5):606-615.

9. Cahill K, Stead LF, Lancaster T. Nicotine receptor partial agonists for smoking cessation. Cochrane Database Syst Rev. 2010;12:CD006103.

10. Civljak M, Sheikh A, Stead LF, Car J. Internet-based interventions for smoking cessation. Cochrane Database Syst Rev. 2010;9:CD007078.

11. Thomsen T, Villebro N, Møller AM. Interventions for preoperative smoking cessation. Cochrane Database Syst Rev. 2010;7:CD002294. 
12. Grimshaw GM, Stanton A. Tobacco cessation interventions for young people. Cochrane Database Syst Rev. 2006;4:CD003289.

13. Rigotti NA, Quinn VP, Stevens VJ, et al. Tobacco-control policies in 11 leading managed care organizations: progress and challenges. Eff Clin Pract. 2002;5:130-136. Error! Hyperlink reference not valid.

14. A clinical practice guideline for treating tobacco use and dependence. US Public Health Service. Tobacco Use and Dependence Clinical Practice Guideline Panel. JAMA. 2000;283:3244-3254.

15. Solberg LI, Boyle RG, Davidson G, Magnan SJ, Carlson CL. Patient satisfaction and discussion of smoking cessation during clinical visits. Mayo Clin Proc. 2001;76:138-143.

16. Toll BA, Martino S, Latimer A, et al. Randomized trial: Quitline specialist training in gain-framed vs standard-care messages for smoking sessation. J Natl Cancer Inst. 2010;102:96-106.

17. Hollis JF, McAfee TA, Fellows JL, Zbikowski SM, Stark M, Riedlinger $\mathrm{K}$. The effectiveness and cost effectiveness of telephone counselling and the nicotine patch in a state tobacco quitline. Tob Control. 2007;16(Suppl 1):i53-i59.

18. An LC, MD, Bluhm JH, Foldes SS, et al. A randomized trial of a pay-for-performance program targeting clinician referral to a state tobacco quitline. Arch Intern Med. 2008;168:1993-1999.

19. Winickoff JP, Friebely J, Tanski SE, et al. Beliefs about the health effects of "thirdhand" smoke and home smoking bans. Pediatrics. 2009; 123(1): e74-e79.

20. World Health Organization Study Group on Tobacco Regulation. Tobreg scientific recommendation: devices designed for the purpose of nicotine to the respiratory system in which tobacco is not necessary for their operation. In: Report on the Scientific Basis of Tobacco Regulation: Third Report of a WHO Study Group. Geneva: World Health Organization; 2009.

21. Ben Schott. Vaping. Puffing on electronic cigarettes. Schott's Vocab. New York Times. July 15, 2010. http://schott.blogs.nytimes. com/2010/07/15/vaping. Accessed January 7, 2011.

22. Lefler D. Ban doesn't include electronic cigarettes. Wichita Eagle. July 6, 2010. http://www.kansas.com/2010/07/06/1391740/ban-doesnt include-electronic.html\#. Accessed January 7, 2011.

23. Yamin CK, Bitton A, Bates DW. E-cigarettes: a rapidly growing internet phenomenon. Ann Intern Med. 2010;153:607-609.

24. Westenberger BJ. US Food and Drug Administration evaluation of e-cigarettes. Center for Drug Evaluation and Research, Division of Pharmaceutical Analysis. Rockville, MD: US Food and Drug Administration, 2009. http://www.fda.gov/downloads/Drugs/ScienceResearch/ UCM173250.pdf. Accessed January 7, 2011.

25. Leondiadis L. Results of chemical analyses in NOBACCO electronic cigarette refills. Athens, Greece: Mass Spectrometry and Dioxin Analysis Laboratory, National Centre for Scientific Research ("Demokritos"), 2009.

26. Flouris AD, Oikonomou DN. Electronic cigarettes: miracle or menace? BMJ. 2010;340:c311. doi:10.1136/bmj.c311.

27. Laugesen M, Thornley S, McRobbie H, Bullen C. How safe is an e-cigarette? The results of independent chemical and microbiological analysis. Christchurch, New Zealand: Health New Zealand, 2008. http://www.healthnz.co.nz/Portland2008ECIG.pdf. Accessed January 7, 2011.
28. Pauly J, Li Q, Barry MB. Tobacco-free electronic cigarettes and cigars deliver nicotine and generate concern. Tob Control. 2007;16:357.

29. Trtchounian A, Williams M, Talbot P. Conventional and electronic cigarettes (e-cigarettes) have different smoking characteristics. Nicotine Tob Res. 2010;12:905-912.

30. Etter JF. Electronic cigarettes: a survey of users. BMC Public Health. 2010;10:231.

31. Bullen C, McRobbie H, Thornley S, Glover M, Lin R, Laugesen M. Effect of an electronic nicotine delivery device (e cigarette) on desire to smoke and withdrawal, user preferences and nicotine delivery: randomised cross-over trial. Tob Control. 2010;19:98-103.

32. US Food and Drug Administration. FDA Warns of Health Risks Posed by E-cigarettes. FDA Consumer Health information, July 2009. http://www.fda.gov/downloads/ForConsumers/ConsumerUpdates/ UCM173430.pdf. Accessed January 7, 2011.

33. Schick S. Thirdhand smoke: here to stay. Tob Control. 2011;20:1-3.

34. Matte GE, Quintana PJ, Zakarian JM, et al. When smokers move out and non-smokers move in: residential thirdhand smoke pollution and exposure. Tob Control. 2011;20:1-3.

35. Becquemin MH, Bertholon JF, Bentayeb M, et al. Thirdhand smoking: indoor measurements of concentration and sizes of cigarette smoke particles after resuspension. Tob Control. 2010;19:347-348.

36. Kraev TA, Adamkiewicz G, Hammond SK, Spengler JD. Indoor concentrations of nicotine in low-income, multi-unit housing: associations with smoking behaviours and housing characteristics. Tob Control. 2009; 18:438-444

37. Ciuk J, Volkmer RE, Edwards JW. Domestic nitrogen oxide exposure, urinary nitrate, and asthma prevalence in preschool children. Arch Environ Health. 2001;56(5):433-438.

38. Leaderer BP, Naeher L, Jankun T, et al. Indoor, outdoor, and regional summer and winter concentrations of PM10, PM2.5, SO4(2)-, H+, $\mathrm{NH} 4+, \mathrm{NO} 3-, \mathrm{NH} 3$, and nitrous acid in homes with and without kerosene space heaters. Environ Health Perspect. 1999;107(3): 223-231.

39. Sleimana M, Gundela LA, Pankowb JF, Jacob P, Singera BC, Destaillats H. Formation of carcinogens indoors by surface-mediated reactions of nicotine with nitrous acid, leading to potential thirdhand smoke hazards. PNAS. 2010;107:6576-6581. doi:10.1073/pnas.0912820107.

40. Fortmann AL, Romero RA, Sklar M, et al. Residual tobacco smoke in used cars: futile efforts and persistent pollutants. Nicotine Tob Res. 2010;12:1029-1036.

41. Hecht SS. Tobacco carcinogens, their biomarkers and tobacco-induced cancer. Nat Rev Cancer. 2003;3:733-744.

42. Petrick L, Destaillats H, Zouev I, Sabach S, Dobowski Y. Sorption, desorption, and surface oxidative fate of nicotine. Phys Chem Chem Phys. 2010;12:10356-10364.

43. Petrick LM, Svidovsky A, Dubowski Y. Thirdhand smoke: heterogeneous oxidation of nicotine and secondary aerosol formation in the indoor environment. Environ Sci Technol. 201;45:328-333.

44. Tuma RS. Thirdhand smoke: studies multiply, catchy name raises awareness. J Natl Cancer Inst. 2010;102:1004-1005.

45. Dreyfuss JH. Thirdhand smoke identified as potent, enduring carcinogen. CA Cancer J Clin. 2010;60:203-204.
International Journal of General Medicine

\section{Publish your work in this journal}

The International Journal of General Medicine is an international, peer-reviewed open-access journal that focuses on general and internal medicine, pathogenesis, epidemiology, diagnosis, monitoring and treatment protocols. The journal is characterized by the rapid reporting of reviews, original research and clinical studies across all disease areas.

\section{Dovepress}

A key focus is the elucidation of disease processes and management protocols resulting in improved outcomes for the patient.The manuscript management system is completely online and includes a very quick and fair peer-review system. Visit http://www.dovepress.com testimonials.php to read real quotes from published authors. 